OPEN ACCESS

Edited by:

Cristina Mammucari,

University of Padova, Italy

Reviewed by:

Manuela Côrte-Real,

University of Minho, Portugal

Vito De Pinto,

University of Catania, Italy

*Correspondence:

Silvia Campello

silvia.campello@uniroma2.it

Specialty section:

This article was submitted to Molecular and Cellular Oncology,

a section of the journal

Frontiers in Oncology

Received: 26 January 2017

Accepted: 11 April 2017

Published: 02 May 2017

Citation:

Bordi M, Nazio F and Campello S

(2017) The Close Interconnection

between Mitochondrial Dynamics and

Mitophagy in Cancer.

Front. Oncol. 7:81.

doi: 10.3389/fonc.2017.00081

\section{The Close Interconnection between Mitochondrial Dynamics and Mitophagy in Cancer}

\author{
Matteo Bordi', Francesca Nazio ${ }^{2}$ and Silvia Campello ${ }^{1,3 *}$ \\ ${ }^{1}$ Department of Biology, University of Rome Tor Vergata, Rome, Italy, ${ }^{2}$ Department of Pediatric Hematology and Oncology, \\ IRCCS Bambino Gesù Children's Hospital, Rome, Italy, ${ }^{3}$ IRCCS, Fondazione Santa Lucia, Rome, Italy
}

Recent decades have revealed the shape changes of mitochondria and their regulators to be main players in a plethora of physiological cell processes. Mitochondria are extremely dynamic organelles whose highly controlled morphological changes respond to specific and diverse pathophysiological needs. Thus, their qualitative control is crucial for the determination of cell function and fate. Moreover, ever-new metabolic changes, mainly attributable to mitochondrial (dys)functions, are strongly connected to cancer and its microenvironment. For this reason, the aspects controlling mitochondria activity and status are in the oncological spotlight. In this review, we elucidate the most intriguing discoveries related to two apparently independent but strictly interconnected processes crucial for the organelle functionality and fate, mitochondrial dynamics, and mitophagy. We will mostly focus on their metabolic interconnections and regulations that can causally foster a tumoral context.

Keywords: cancer, mitochondrial dynamics, autophagy, mitophagy, mitochondrial fate

\section{THE MITOCHONDRIA BALLET}

A large body of evidence has highlighted the existence of a close interconnection between the cancer cells fate and the two apparently unrelated cellular processes of mitochondrial dynamics and autophagy. Indeed, cancer cells regulate the morphology of their mitochondria on the basis of their bioenergetic and biosynthetic needs to sustain proliferation and migration, and to escape apoptosis. The modulation of the organelles shape is also crucial for the qualitative control of the same, which mainly depends on the selective autophagic process called mitophagy.

Mitochondria are highly dynamic organelles, forming an active network capable of undergoing sudden changes to adapt its structure to the energetic and physiological needs of the cell (1). Mitochondria shape results from a balance between fusion and fission mechanisms in response to endogenous and exogenous stimuli or insults (2). These organelles are fundamental for: (i) energy production through an efficient oxidative phosphorylation (OXPHOS) and ATP production and (ii) biosynthetic metabolism and production of metabolites (3). On the other hand, mitochondria are the major source of reactive oxygen species (ROS) that could cause oxidative damage to proteins, lipids, and DNA (4) and result in aging and several diseases, including neurodegenerative disease, diabetes, and cancer $(5,6)$ see Figure 1. 


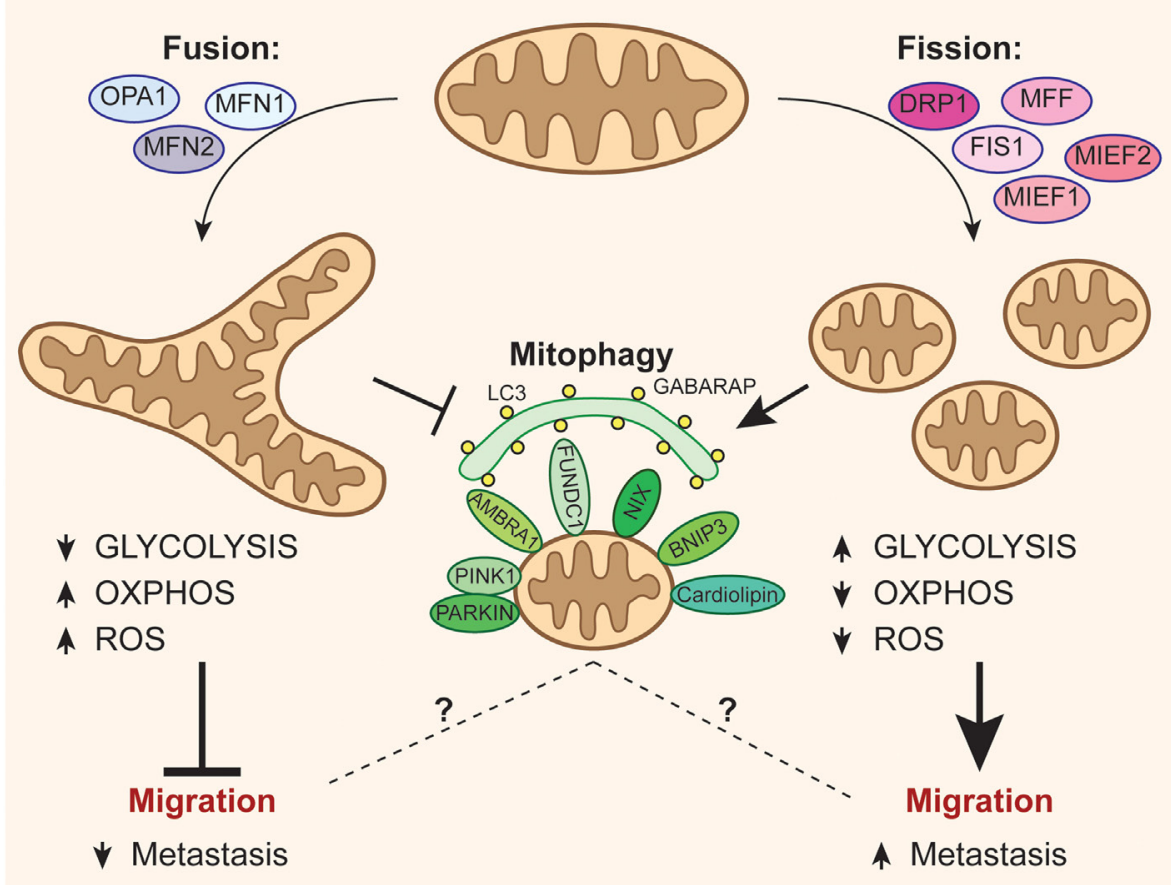

FIGURE 1 | Mitochondrial dynamics and fate can affect metastatization of cancer cells. The activation of optic atrophy 1-mitofusin (MFN)1/2-mediated mitochondrial fusion promotes oxidative phosphorylation (OXPHOS) at the expense of anaerobic glycolysis, leading to increased reactive oxygen species (ROS) levels and, consequently, disfavoring the migration of cancer cells. On the other hand, mitochondrial fission events mediated by dynamin-related protein-1 (DRP1) recruitment through its accessory proteins (mitochondrial fission factor, FIS1, MIEF1/2) inhibit oxidative metabolism by increasing the energetic yield of glycolysis, and reduces ROS production, so easing the process of metastasis. Moreover, fission is critical for the correct activation of mitophagy, even though it remains ambiguous how mitophagy may be related to tumor migration.

\section{Mitochondrial Fusion Dynamism}

In the fusion phase, the outer and inner mitochondrial membranes (OMM and IMM, respectively) of two distinct mitochondria fuse with each other, so resulting in the mixing of the mitochondrial content (7). Fusion is regulated by the activity of dynamin-related GTPases Mitofusin (MFN) 1 and 2, and by optic atrophy 1 (OPA1) (1). Upon GTP binding, the GTPase domains of MFN1 and MFN2 interact, forming homo- and hetero-dimers that undergo conformational changes upon GTP hydrolysis, bringing the opposing OMM into close contact to foster their fusion (8-11). OPA1, instead, facing the inner membrane space, regulates the IMM fusion (1), together with MFN1 (12). The pleiotropic OPA1 is also involved in mitochondrial cristae architecture, mitochondrial bioenergetics, and apoptosis (13-15). Distinctive OPA1 isoforms have been identified, derived by differential RNA splicing and precursor protein processing (16) [for more details, see Kasahara and Scorrano (1)]. Moreover, OPA1 activity is regulated by a proteolytic cascade; the long isoform, i.e., characterized by pro-fusion activity, can be cleaved by: the large intermembrane space AAA-protease Yme1L $(17,18)$, the mitochondrial matrix ATPase proteases (19), the metalloprotease paraplegin $(20)$, and $\operatorname{OMA} 1(19,21)$. The shorter isoform is a substrate for the presenilin-associated rhomboid-like (PARL) and contributes to regulate the cristae morphology and apoptosis (1). It is possible that tissue-specific expression of OPA1-cleaving proteases and/or splice variants contributes to the complex pattern of OPA1 processing. Fusion processes are inhibited upon specific stress stimuli (such as mitochondrial uncoupling agents like CCCP), promoting the activation of the opposite fission pathway; when mitochondria lose membrane potential $[\Delta \Psi(\mathrm{m})]$ or the ATP levels decrease, OMA1 is stabilized and enhances the cleavage of OPA1 so preventing inner membrane fusion (21). As the long and short OPA1 isoforms are both required for fusion, complete conversion of OPA1 to the short isoform by OMA1 shuts off the fusion machinery of dysfunctional mitochondria. This activity could contribute to a quality control process by preventing fusion of defective mitochondria. Under the same circumstances, fusion can be prevented by MFNs degradation mediated by both PTEN-induced putative kinase 1 (PINK1) and PARKIN during mitophagy, a selective removal of damaged mitochondria by macroautophagy (described in detail in the next section) (22). Likewise, in response to genotoxic stresses, MFN2 is phosphorylated by Jun $\mathrm{N}$-terminal kinase (JNK), ubiquitinated by the E3 ubiquitin ligase HUWE1 and consequently degraded by the proteasome, leading to mitochondrial fragmentation and apoptotic cell death (23).

\section{Mitochondrial Fission Dynamism}

As mentioned above, when fusion is inhibited, fission is initiated. Fission is mediated by the activation of dynamin-related 
protein-1 (DRP1), a protein mainly localized in the cytosol that cycles between the cytosol and the OMM, where it constricts and cuts the organelles (16).

Many pathways regulating the activities of the fission machinery in mammalian cells have been discovered. Also DRP1 activity is reversibly regulated by several post-translational modifications, in particular by phosphorylation/dephosphorylation events. The phosphatase calcineurin dephosphorylates DRP1 on Ser637 (24) leading to its translocation to OMM, where it interacts with different accessory factors such as the mitochondrial fission factor (MFF), FIS1, Mid49/Mief1, and Mid51/Mief2 that may act in various ways to promote DRP1-dependent fission $(16,25)$. Once on the OMM, DRP1 assembles into multimeric spiralic structures and, through hydrolysis of GTP, mediates constriction of the spiral and scission in two distinct mitochondria (16, 26). Three protein kinases that phosphorylate different DRP1 serine residues have been identified: (1) high-glucose-induced $\mathrm{Ca}^{2+}$, extracellular signal-regulated kinase 1/2 (ERK1/2) causing mitochondrial fission (27); (2) the protein kinase A (PKA) that inhibits DRP1 GTPase activity and therefore prevents fission (28); and (3) a kinase anchoring protein 1 (AKAP1), which upon hypoxia conditions stabilizes PKA on neurons OMM, so exacerbating PKA negative regulation of DRP1 and promoting both mitochondrial elongation and neuronal survival (29). Opposed to PKA/AKAP1, PP2A/B $\beta 2$-mediated dephosphorylation of Drp1 enhances fission, so regulating neuronal development via mitochondrial bioenergetics (30). During mitosis, CDK1/cyclin $\mathrm{B}$ leads to mitochondrial fragmentation activating DRP1 via its phosphorylation at Ser616, thereby facilitating an appropriate distribution of mitochondria to daughter cells (31). Furthermore, it has been described that the E3 ligase PARKIN (32) and another E3 ligase, MARCH-V (33), mediate the degradation of DRP1 via proteasome, while positively modulating MFN2, probably thus boosting fusion. Mitochondrial-anchored protein ligase is a mitochondrion-anchored small ubiquitin-like modifier (SUMO) ligase that sumoylates DRP1 to stimulate mitochondrial fission (34). Removal of SUMO from DRP1 involves sentrin-specific protease 5 (SENP5), a SUMO protease that recognizes several mitochondrial targets (35). Despite many pieces of the mitochondrial dynamics machinery puzzle being now in place, further pieces will probably be discovered and need to be added to better explain the role of all the components regulating the fusion and fission pathways.

\section{Mitochondrial Dynamics-Related Modulation of Mitochondria Quality versus Efficiency}

The mitochondrial shape remodeling is intimately connected to the fate of the organelles; in fact, the mitochondrial function is maintained by the coordinated activation of the mitochondrial quality control pathway that guarantees the degradation of damaged mitochondria, so safeguarding the cell from the activation of apoptosis (1). The selective removal of damaged mitochondria is ensured by macroautophagy (hereafter referred to as autophagy): it is an evolutionarily conserved process, which triggers degradation of bulk cytoplasm, long-lived proteins, and entire organelles via lysosome, for recycling purposes $(36,37)$. Autophagy is upregulated during cellular stress (38) (increase of oxidative stress, ER-stress, DNA damage) and is closely linked to the mechanisms underlying aging (39). Autophagy is a very dynamic process described as a flux (40). The "autophagy core complex," a multimolecular machinery including the ULK1 complex and the BECN1-VPS34 complex, initiates the formation of a double-membrane vesicle, termed autophagosome (AP), which surrounds the substrates (37); the matured AP is thus delivered to lysosomes for degradation (41). Several kinase complexes, including MTOR complex 1 that negatively affects ULK1 activity (42), tightly regulate each step of autophagy (36). Whereas the autophagy response to starvation is bulk degradation of cytosolic material, other types of stress, such as damaged organelles or aggregated proteins (39), require selective sequestration of the specific cargo into the autophagosomal membranes. Selectivity is achieved through autophagy receptors, such as SQSTM1/p62, NBR1, NDP52, OPTINEURIN (OPTN), TAX1BP1, and NIX, which recognize, on the one hand, cargos tagged by degradation signals and, on the other hand, the autophagosomal membrane through their LC3-interacting regions (LIR) $(43,44)$. The specific autophagic breakdown of mitochondria is termed mitophagy (45). In this case, mitochondria with decreased membrane potential are less likely to be engaged in subsequent fusion events and, instead, are prone to be removed through mitophagy. By contrast, inhibition of fission impedes mitophagy and results in decline of the respiratory capacity, whereas, arrest of autophagy leads to the accumulation of mitochondria with low membrane potential and low OPA1 (46). Thus, as already mentioned, mitophagy and mitochondrial dynamics are tightly interconnected. In recent years, distinct mitophagic pathways have been identified, revealing that the elimination of altered mitochondria is a critical step for mammalian cell fate.

\section{Spotlight on the Molecular Pathways}

PTEN-induced putative kinase 1 (PINK1)-PARKIN-dependent mitophagy is the most characterized mitophagic pathway. In basal healthy conditions, PINK1 is constitutively cleaved by the mitochondrial processing protease (MPP) and, further, cleaved by PARL and so rapidly degraded $(47,48)$. Upon mitochondria membrane depolarization, MPP and PARL are inhibited and PINK1 is consequently stabilized at the $\operatorname{OMM}(49,50)$; this leads to its autophosphorylation $(50,51)$ and to the phosphorylation of its substrates that initiates mitophagy. In particular, PINK1 phosphorylates ubiquitin at its Ser65; this leads to the activation and recruitment to mitochondria of the E3 ligase PARKIN (52-54), thus amplifying the signal started by PINK1 (55). Fascinatingly, a recent study shows that, in HeLa cells, PINK1 phosphorylates and also recruits NDP52 and OPTINEURIN, two autophagic cargo receptors that, despite being considered downstream of AP formation, are important for re-localization of ULK1 on damaged mitochondria (55). Moreover, as previously mentioned, PINK1 mediates the phosphorylation of MFN2 that acts as a relevant receptor for a correct PARKIN translocation (56).

Following its translocation to the mitochondrial surface, PARKIN modifies many OMM proteins by both K48- and K63linked ubiquitin chains (57-59). On the one hand, PARKIN 
promotes proteasome-degradation of MFN1, MFN2, some TOM complex proteins (TOM20) (22), and mitochondrial rhoGTPase-1 (MIRO1) (60), so favoring the mitochondrial fission and arresting mitochondria motility; on the other hand, PARKIN mediates K63-linked ubiquitination of substrates such as VDAC1, a mitochondrial protein voltage-dependent anion channel, which acts as a signal for recruiting autophagic cargo receptors $(22,61,62)$. A very recent publication has opened new insights into the mitophagy mechanisms, revealing that an IMM protein prohibitin 2 (PHB2) acts as a mitophagy receptor directly binding to LC3 through its LIR motif and is essential for PARKIN-mediated mitophagy (63).

Recruitment of PARKIN to depolarized mitochondrial membranes is inhibited by the anti-apoptotic proteins BCL-XL, Mcl-1, and BCL2, in a BECLIN1-independent manner $(64,65)$. Additionally, the contribution of deubiquitinating enzymes (DUBs) on the PINK1/PARKIN-mediated mitophagy has been characterized. DUBs regulate ubiquitin signals by removing or trimming ubiquitin linkages, and thus they can play important roles in modulating mitophagy. The OMM-localized DUB ubiquitin-specific processing proteases USP30 (66) and USP15 (67) antagonize PARKIN activity by cleaving ubiquitin chains on mitochondria (22). USP8, instead, antagonizes mitophagy by directly deubiquitinating PARKIN, so contributing to its recruitment and activity (68). Moreover, Heo et al. report that mitochondrial damage activates the kinase TBK1, which phosphorylates autophagy adaptors OPTN, NDP52, and SQSTM1/ p62, so enhancing their recruitment to damaged mitochondria to promote mitophagy (44). Collectively, these events constitute a feedforward amplification mechanism to endorse mitophagy $(44,58)$.

Although PINK1-PARKIN-dependent mitophagy is the most characterized and most tumors-linked pathway, as mentioned, alternative pathways have been recently described, and different cell types might have different degrees of sensitivity for the activation of characteristic mitophagic pathways. For instance, PARKIN is further reported to interact with Activating molecule in BECN1-regulated autophagy protein 1 (AMBRA1) that activates BECLIN1 complex, endorsing the AP formation nearby (69). However, AMBRA1 can also induce mitophagy regardless of PARKIN and PINK1, via a direct interaction with LC3 (70) and allegedly, via its positive loop on ULK1 (42). In fact, a recent study points out that, upon mitophagy induction by either hypoxia or mitochondrial uncouplers, ULK1 translocates to mitochondria and phosphorylates the cargo receptor FUN14 domain-containing protein, so regulating mitophagy (71). In neurons, pro-mitophagy stimuli cause the translocation of the phospholipid cardiolipin from IMM to OMM, thus favoring the recognition of damaged mitochondria by the autophagic machinery in a PINK1-PARKIN-independent way (72). Intriguingly, Toyama et al. describe that energy-sensing adenosine monophosphate (AMP)-activated protein kinase (AMPK), a positive regulator of ULK1 that activates autophagy for maintaining the energy homeostasis, triggers fission activation, and consequently mitophagy, through phosphorylation of MFF, thus unveiling a new central role for AMPK in the regulation of mitochondria homeostasis (73).
Previous studies have also identified the $\mathrm{BH} 3$-only protein NIX (Bnip3L) and BCL2/adenovirus E1B $19 \mathrm{kDa}$ proteininteracting protein 3 (BNIP3) as mitophagy receptors due to their direct interaction with LC3 (74); during the maturation of erythroid cells, NIX is involved in programmed removal of mitochondria (75), while both BNIP3 and NIX are key players in hypoxia-induced mitophagy $(74,75)$. In addition to mitophagy, a new pathway for mitochondrial quality control has been recently discovered (76) in which PINK1-PARKIN drive the formation of mitochondrial-derived vesicles for the delivery of mitochondrial oxidized proteins to lysosomes [reviewed by Sugiura et al. (76)].

\section{Mitochondria in Cancer: A Question of Balance between Energy Production and Clearance}

Recent publications have identified a linear relation between mitochondria fate and cancer (77-81). In fact, mitochondria can be connected to cancer formation and progression, and their contribution has a strong impact on invasiveness and metastatic profile of cancers (79). A growing body of evidence suggests that many cancer cell lines and solid tumors undergo a drastic metabolic reprogramming; they limit the tricarboxylic acid cycle (TCA) and mitochondrial OXPHOS as a consequence of mutations that affect the activity of TCA key enzymes or the activity of OXPHOS complexes $(77,82)$. Therefore, cancer cells veer toward a prominent use of glycolysis as the main source for ATP production ("Warburg effect") (83), so upregulating glucose uptake [reviewed by Gaude and Frezza (81)]. However, the connection between mitochondria dysfunction and cancer is not just related to metabolism (83). In fact, mitochondria activities can also directly or indirectly affect nuclear or mitochondrial DNA expression (84) and mutations (81), epigenetic changes (such as methylation) (77), cell migration (78), and cell death (85). Moreover, an imbalance in the mitochondrial degradation process results in progressive alteration of cellular homeostasis, which, in turn, may set the stage for the development of tumor cells. This suggests the existence of an intricate reciprocal interplay between mitochondria, autophagy/mitophagy, and tumor initiation. Autophagy has always been perceived as playing a double-faceted role in tumorigenesis, either supporting survival or promoting death, depending on the different cellular contexts. For instance, mice with heterozygotic deletion of Becn1 are susceptible to spontaneous tumors, while deletion of other key autophagic genes such as Atg5 or Atg7 leads to the appearance of only benign liver tumors (86). By contrast, some types of tumors are dependent on the activation of autophagy, such as RASdriven cancers $(86,87)$. In other cases, tumor cells can increase autophagy to promote chemo- and radio-resistance (88).

Intriguingly, autophagy has been described as being critical for innate and adaptive immunity through regulating antigens processing and presentation (89); moreover, autophagy induction helps the host immune system to properly recognize and eliminate pre-malignant and malignant cells (90). Recently, Pietrocola and collaborators have demonstrated that short-term fasting or autophagy-inducing caloric restriction mimetics enhances 
anticancer immune responses by activating immune effector $\mathrm{T}$ lymphocytes, thus preventing cancer cells escaping from immuno-surveillance (91). This activation of autophagy, in cancer cells, leads to their ATP release into the extracellular space where ATP acts as a chemotactic factor attracting T cells and, thus, favoring tumor growth reduction (91). Considering that mitochondria elongation correlates with more performing mitochondria and more cristae that should guarantee a sustained ATP production (92), we speculate that, in Pietrocola's system mitochondrial dynamics might have a role. Thus, ideally, by pharmacologically modulating mitochondrial dynamics of tumor cells, we might act on and improve anticancer immuno-surveillance. These assumptions reinforce the belief that mitochondrial dynamics and autophagy/mitophagy are coevolutionarily interconnected along all the cell types.

In line with these notions, increasing evidence interestingly also links dysfunctions in mitophagy to cancer development $(65,76$, $80,93)$, even though how they are connected it is strictly dependent on the cancer type. A pathological decrease of mitophagy efficiency causes accumulation of damaged mitochondria. This leads to disruption of redox balance and to an increase of detrimental oxidative damage, such as ROS-induced DNA mutations; hence, the increase in free radicals production raises the possibility of tumors developing due to genetic instability $(94,95)$. Conversely, mitophagy can protect cells from apoptosis and promote tumor cell survival under some adverse conditions $(71,96)$.

PARKIN deletions or loss of function mutations have been identified in ovarian, breast, bladder, and lung cancers (this topic has recently been thoroughly reviewed elsewhere) (97); additionally, mice null for PARKIN develop spontaneous macroscopic hepatic tumors (98). Mutation in PARK6 gene (PINK1) (99) has been observed in neuroblastoma, so raising the hypothesis that alteration of mitophagy may have a causal role in certain tumors. As described above, AMBRA1 is a PARKIN-interacting protein indispensable for the final step of PARKIN-triggered mitophagy (70). Ambral heterozygosity, in mice, has been recently associated to tumorigenesis, this gene thus acting as a tumor suppressor gene (100). Although the exact role of AMBRA1 in cancer insurgence is still largely unclear, one of the possible mechanisms, proposed by Strappazzon and Cecconi, hypothesizes that AMBRA1 dysregulation in mitophagy might be related to carcinogenesis (65). Moreover, increase in ROS levels, in particular nitric oxide levels, further impairs PARKIN E3 ligase activity (101), thus exacerbating the accumulation of damaged mitochondria, on a feedback loop. On the other hand, mitophagy is triggered by mild oxidative stress through HIF-1 $\alpha$ (102), a transcriptional factor normally activated during hypoxic conditions, which positively regulates the expression of BNIP3 and NIX, these thus acting as tumor suppressors (93).

As already mentioned above, some tumors can use mitophagy to escape cell death activation, or as an adaptive mechanisms during the first stages of solid tumor development, when cancer cells are in the typical hypoxia environment $(71,96,103)$. In fact, K-ras-induced lung tumors require mitophagy to sustain mitochondrial function and lipid catabolism and, in this case, inhibition of mitophagy leads to proliferative arrest, so negatively altering the tumor fate (104). In addition, a recent study indicates that AMPK confers metabolic stress resistance on leukemiainitiating cells and promotes leukemogenesis by suppressing ROS production and maintenance of metabolism (105), this probably also through mitophagy activation.

Interestingly, it has been demonstrated that cancer cells can act as metabolic parasites and extract nutrients from host cells by inducing catabolic processes (autophagy, mitophagy, aerobic glycolysis, and lipolysis) (106). In these cases, fibroblasts adjacent to the tumor (cancer-associated fibroblasts) positively regulate autophagy and mitophagy in order to support cancer cells metabolic needs for growth, proliferation, migration, and invasion (106-108). This demonstrates that tumor cells are able to coordinate these two pathways in different ways as a protective mechanism to ensure their own survival.

In this context, also some OMM proteins play a fundamental role in driving and promoting cancer cell progression. Recently, VDAC1, a voltage-dependent anion channel (109, 110), contributes to the metabolic phenotype of cancer cells regulating mitochondrial activity and glucose metabolism. In fact, VDAC1 directly binds to hexokinase II (HK II), so endorsing its activity (111). HK is an enzyme that catalyzes the first reaction of glycolysis, which is upregulated in broad variety of tumor types sustaining elevated rate of glucose catabolism and consequently rapid growth rates (111). Moreover, the interaction between VDAC1 and HK II inhibits mitochondrial-induced apoptosis, so helping tumor cells to elude cell death (110); furthermore, HK dissociation from mitochondria can be also a strong proapoptotic stimulus independent from VDAC1 (112). Though, VDAC1 is over-expressed in many cancer types, and its silencing inhibits tumor development (113). Furthermore, as described above, VDAC1 is a mitochondrial target of PARKIN, required for its efficient targeting to damaged mitochondria (62) and, therefore, essential for PINK1/PARKIN-mediated mitophagy (61). This demonstrates that, beside fusion and fission proteins, other OMM proteins have a role in the intricate interconnection between mitochondria fate and cancer.

\section{Mitochondrial Dynamics Are Crucial for Tumor Cell Fate Decision}

Hitherto, we have discussed the role of mitophagy regulation in the progression of cancer, highlighting some link between this process and mitochondrial dynamics. But which is the actual effect of mitochondrial dynamics in tumor? Like a healthy cell, a cancer cell can undergo mitochondrial morphology changes for responding to the external environment. This decision is critical for cancer cell fate determination during tumor progression see Figure 1. In fact, cancer cells might undergo extensive fusion to promote respiratory capacity, to support proliferation, or to promote cell survival in adverse conditions, such as upon anticancer treatment (114) or glucose deprivation (115), for escaping apoptosis activation. On the other hand, cancer cells might foster fission to repress oxidative metabolism, preventing ROS formation and oxidative damage, or most likely to encourage activation of mitophagy, as mentioned. In other cases, tumor cells rely on glutamine-dependent reductive carboxylation. They upregulate mitochondrial biogenesis and increase mitochondrial respiratory chain capacity through the activation of the potent 
oncogene C-MYC, which, in turn, regulates the mitochondrial network controlling the expression of multiple mitochondrial genes $(116,117)$. From a molecular viewpoint, and related to tumor progression and severity, more than its onset, alteration of DRP1 activity seems to be a common feature of many cancer cells that, therefore, exhibit fragmented mitochondria (78, 118-123). In pancreatic cancer, DRP1 activation by ERK2 and mitochondrial fission are crucial for malignancies (119). In brain tumor initiating cells, cyclin-dependent kinase 5 (CDK5)-mediated DRP1 activation drives massive mitochondrial fragmentation, affects AMPK pathway, and correlates with poor prognosis (123). In lung cancer cells, increased mitochondrial fission is due to an upregulation of DRP1 expression and an increased ratio of Ser616-to-Ser637 phosphorylation, paralleled by a decrease of MFN2 levels, and impaired fusion. Reverting the DRP1/MFN2 imbalances result in in vitro reduction of cancer cell proliferation and activation of apoptosis (118). No less importantly, the coordination between mitochondrial dynamics and mitosis (so-called mitotic fission), which ensures equitable distribution of mitochondria to daughter cells, plays a role in cancer, clearly related to the requirement for mitochondrial division during mitosis. This phenomenon is regulated by cyclin B1-CDK1, which simultaneously initiates mitosis and activates DRP1 by phosphorylating its Ser616 (121). Another mitotic kinase, Aurora A, phosphorylates the Ras-like GTPase (RALA), leading to mitotic mitochondrial accumulation of RALA and its effector, ralA binding protein 1 (RALBP1). RALBP1 acts as a scaffold for recruiting DRP1 and cyclin-CDK to mitochondria and inducing fission (31).

DRP1 activation, however, is closely linked to migration and invasion of tumor cells. It has been described that imbalance of the mitochondria phenotype toward fragmentation coordinates the migratory capability in cells where migration represents a crucial physiological function, such as T lymphocytes (124). Indeed, ample data have started to highlight a strict correlation between the levels of DRP1-mediated mitochondrial fission and diverse metastatic states of different tumoral cells (125); these cells boost mitochondria fission and re-localization up in order to locally fulfill their high-energy requests for migration. This is the case of invasive breast carcinoma cells that require DRP1-dependent mitochondrial fission for re-localizing the organelles to lamellipodial regions where they need to satisfy a localized growing energy demand, so marking this process as a critical early developmental step in metastatic breast cancer (78). DRP1 overexpression is also associated with malignant oncocytic thyroid tumors, positively regulating migration of thyroid cancer cells (120). In glioblastoma cells, DRP1-induced mitochondrial fission and consequent

\section{REFERENCES}

1. Kasahara A, Scorrano L. Mitochondria: from cell death executioners to regulators of cell differentiation. Trends Cell Biol (2014) 24:761-70. doi:10.1016/j. tcb.2014.08.005

2. Campello S, Strappazzon F, Cecconi F. Mitochondrial dismissal in mammals, from protein degradation to mitophagy. BBA Bioenerg (2014) 1837:451-60. doi:10.1016/j.bbabio.2013.11.010

3. Graier WF, Frieden M, Malli R. Mitochondria and $\mathrm{Ca}(2+)$ signaling: old guests, new functions. Pflugers Arch (2007) 455:375-96. doi:10.1007/ s00424-007-0296-1 cell migration are driven by hypoxia through HIF-1 $\alpha$ (122). In line with these observations, the perturbation of MIRO1, which is essential for mitochondrial transport, prevents the redistribution of mitochondria to the anterior of moving epithelial cancer cells, so affecting these cells migratory abilities (126). It is important to note that DRP1 and mitochondria remodeling have a constitutive leading role in the modulation of T cell metabolic shifts, this inducing differentiation of T cells, and it is crucial for the detection and clearance of tumor cells (127) into diverse populations (128). Therefore, the connection between mitochondria fragmentation and tumor development raises questions as to whether alteration of fission-fusion processes may also negatively and specifically affect the T-cell anti-tumor surveillance, thus contributing to malignant tumor prognosis.

\section{CONCLUSION}

In spite of considerable progress in understanding the implications of mitochondrial dynamics and mitophagy in controlling tumor origin and progression, and these two processes emerging cross-links, many key issues remain unresolved. Several lines of evidence indicate that it is quite unreasonable to make a general statement of how autophagy and mitophagy may influence tumor. Likewise, based on emerging findings, it appears that mitochondrial shape regulation plays a critical role in the first steps of tumor and in cancer migration/invasion ability. This breaks new ground in the fundamental understanding of possible mechanisms underlying the intimate interplay between mitochondrial homeostasis and tumors.

Although, it remains to be elucidated also how different aspects of mitochondrial metabolism and dynamics may affect the anti-tumor immune response and what their relative contributions to tumor progression or clearance are. This new concept helps to broaden our knowledge and perspectives regarding tumor cure, making it possible to identify new candidate targets for therapy.

\section{AUTHOR CONTRIBUTIONS}

$\mathrm{MB}$ and FN wrote the manuscript and prepared the figures. SC wrote the manuscript, revised and financed the work.

\section{FUNDING}

This work was funded by the Italian Ministry of Health (GR2011-02351643) to SC and by a grant from Fondazione Roma. MB was supported by "Fondazione Umberto Veronesi” fellowship.

4. Zorov DB, Juhaszova M, Sollott SJ. Mitochondrial reactive oxygen species (ROS) and ROS-induced ROS release. Physiol Rev (2014) 94:909-50. doi:10.1152/physrev.00026.2013

5. Wang $\mathrm{CH}, \mathrm{Wu} \mathrm{SB}, \mathrm{Wu} \mathrm{YT}$, Wei YH. Oxidative stress response elicited by mitochondrial dysfunction: implication in the pathophysiology of aging. Exp Biol Med (Maywood) (2013) 238:450-60. doi:10.1177/1535370213 493069

6. Wallace DC. Mitochondrial function and cancer. Nat Rev Cancer (2012) 12:685-98. doi:10.1038/nrc3365

7. Santel A, Fuller MT. Control of mitochondrial morphology by a human mitofusin. J Cell Sci (2001) 114:867-74. 
8. Koshiba T, Detmer SA, Kaiser JT, Chen H, McCaffery JM, Chan DC. Structural basis of mitochondrial tethering by mitofusin complexes. Science (2004) 305:858-62. doi:10.1126/science.1099793

9. Chen H, Detmer SA, Ewald AJ, Griffin EE, Fraser SE, Chan DC. Mitofusins MFN1 and MFN2 coordinately regulate mitochondrial fusion and are essential for embryonic development. J Cell Biol (2003) 160:189-200. doi:10.1083/ jcb.200211046

10. Formosa LE, Ryan MT. Mitochondrial fusion: reaching the end of mitofusin's tether. J Cell Biol (2016) 215:597-8. doi:10.1083/jcb.201611048

11. Qi Y, Yan L, Yu C, Guo X, Zhou X, Hu X, et al. Structures of human mitofusin 1 provide insight into mitochondrial tethering. J Cell Biol (2016) 215:621-9. doi:10.1083/jcb.201609019

12. Cipolat S, Martins de Brito O, Dal Zilio B, Scorrano L. OPA1 requires mitofusin 1 to promote mitochondrial fusion. Proc Natl Acad Sci U S A (2004) 101:15927-32. doi:10.1073/pnas.0407043101

13. Cogliati S, Enriquez JA, Scorrano L. Mitochondrial cristae: where beauty meets functionality. Trends Biochem Sci (2016) 41:261-73. doi:10.1016/j. tibs.2016.01.001

14. Glytsou C, Calvo E, Cogliati S, Mehrotra A, Anastasia I, Rigoni G, et al. Optic atrophy 1 is epistatic to the core MICOS component MIC60 in mitochondrial cristae shape control. Cell Rep (2016) 17:3024-34. doi:10.1016/j. celrep.2016.11.049

15. Chan DC. Fusion and fission: interlinked processes critical for mitochondrial health. Annu Rev Genet (2012) 46:265-87. doi:10.1146/ annurev-genet-110410-132529

16. van der Bliek A, Shen Q, Kawajiri S. Mechanisms of mitochondrial fission and fusion. Cold Spring Harb Perspect Biol (2013) 5:1-16. doi:10.1101/ cshperspect.a011072

17. Griparic L, Kanazawa T, Van Der Bliek AM. Regulation of the mitochondrial dynamin-like protein OPA1 by proteolytic cleavage. J Cell Biol (2007) 178:757-64. doi:10.1083/jcb.200704112

18. Song Z, Chen H, Fiket M, Alexander C, Chan DC. OPA1 processing controls mitochondrial fusion and is regulated by mRNA splicing, membrane potential, and Yme1L. J Cell Biol (2007) 178:749-55. doi:10.1083/jcb.200704110

19. Ehses S, Raschke I, Mancuso G, Bernacchia A, Geimer S, Tondera D, et al. Regulation of OPA1 processing and mitochondrial fusion by m-AAA protease isoenzymes and OMA1. J Cell Biol (2009) 187:1023-36. doi:10.1083/ jcb.200906084

20. Ishihara N, Fujita Y, Oka T, Mihara K. Regulation of mitochondrial morphology through proteolytic cleavage of OPA1. EMBO J (2006) 25:2966-77. doi:10.1038/sj.emboj.7601184

21. Head B, Griparic L, Amiri M, Gandre-Babbe S, van der Bliek AM. Inducible proteolytic inactivation of OPA1 mediated by the OMA1 protease in mammalian cells. J Cell Biol (2009) 187:959-66. doi:10.1083/jcb.200906083

22. Pickrell AM, Youle RJ. Review the roles of PINK1, PARKIN, and mitochondrial fidelity in Parkinson's disease. Neuron (2015) 85:257-73. doi:10.1016/j. neuron.2014.12.007

23. Leboucher GP, Tsai YC, Yang M, Shaw KC, Zhou M, Veenstra TD, et al. Stress-induced phosphorylation and proteasomal degradation of mitofusin 2 facilitates mitochondrial fragmentation and apoptosis. Mol Cell (2012) 47:547-57. doi:10.1016/j.molcel.2012.05.041

24. Cereghetti GM, Stangherlin A, Martins de Brito O, Chang CR, Blackstone C, Bernardi P, et al. Dephosphorylation by calcineurin regulates translocation of DRP1 to mitochondria. Proc Natl Acad Sci U S A (2008) 105:15803-8. doi:10.1073/pnas.0808249105

25. Losón OC, Song Z, Chen H, Chan DC. Fis1, MFF, MiD49, and MiD51 mediate DRP1 recruitment in mitochondrial fission. Mol Biol Cell (2013) 24:659-67. doi:10.1091/mbc.E12-10-0721

26. Antonny B, Burd C, De Camilli P, Chen E, Daumke O, Faelber K, et al. Membrane fission by dynamin: what we know and what we need to know. EMBO J (2016) 35(21):2270-84. doi:10.15252/embj.201694613

27. Yu T, Jhun BS, Yoon Y. High-glucose stimulation increases reactive oxygen species production through the calcium and mitogen-activated protein kinase-mediated activation of mitochondrial fission. Antioxid Redox Signal (2011) 14:425-37. doi:10.1089/ars.2010.3284

28. Chang C-R, Blackstone C. Cyclic AMP-dependent protein kinase phosphorylation of DRP1 regulates its GTPase activity and mitochondrial morphology. J Biol Chem (2007) 282:21583-7. doi:10.1074/jbc.C700083200
29. Merrill RA, Dagda RK, Dickey AS, Cribbs JT, Green SH, Usachev YM, et al. Mechanism of neuroprotective mitochondrial remodeling by PKA/AKAP1. PLoS Biol (2011) 9:e1000612. doi:10.1371/journal.pbio.1000612

30. Dickey AS, Strack S. PKA/AKAP1 and PP2A/B 2 regulate neuronal morphogenesis via DRP1 phosphorylation and mitochondrial bioenergetics. J Neurosci (2011) 31:15716-26. doi:10.1523/JNEUROSCI.3159-11.2011

31. Kashatus DF, Lim KH, Brady DC, Pershing NL, Cox AD, Counter CM. RALA and RALBP1 regulate mitochondrial fission at mitosis. Nat Cell Biol (2011) 13:1108-15. doi:10.1038/ncb2310

32. Wang H, Song P, Du L, Tian W, Yue W, Liu M, et al. Parkin ubiquitinates DRP1 for proteasome-dependent degradation: implication of dysregulated mitochondrial dynamics in Parkinson disease. J Biol Chem (2011) 286:11649-58. doi:10.1074/jbc.M110.144238

33. Karbowski M, Neutzner A, Youle RJ. The mitochondrial E3 ubiquitin ligase MARCH5 is required for DRP1 dependent mitochondrial division. J Cell Biol (2007) 178:71-84. doi:10.1083/jcb.200611064

34. Harder Z, Zunino R, McBride H. SUMO1 conjugates mitochondrial substrates and participates in mitochondrial fission. Curr Biol (2004) 14:340-5. doi:10.1016/j.cub.2004.02.004

35. Zunino R, Schauss A, Rippstein P, Andrade-Navarro M, McBride HM. The SUMO protease SENP5 is required to maintain mitochondrial morphology and function. J Cell Sci (2007) 120:1178-88. doi:10.1242/jcs.03418

36. Abada A, Elazar Z. Getting ready for building: signaling and autophagosome biogenesis. EMBO Rep (2014) 15:839-52. doi:10.15252/embr.201439076

37. Bernard A, Klionsky DJ. Autophagosome formation: tracing the source. Dev Cell (2013) 25:116-7. doi:10.1016/j.devcel.2013.04.004

38. Pietrocola F, Izzo V, Niso-Santano M, Vacchelli E, Galluzzi L, Maiuri $\mathrm{MC}$, et al. Regulation of autophagy by stress-responsive transcription factors. Semin Cancer Biol (2013) 23:310-22. doi:10.1016/j.semcancer.2013. 05.008

39. Nixon RA. The role of autophagy in neurodegenerative disease. Nat Med (2013) 19:983-97. doi:10.1038/nm.3232

40. Klionsky DJ, Abdelmohsen K, Abe A, Abedin MJ, Abeliovich H, Acevedo Arozena A, et al. Guidelines for the use and interpretation of assays for monitoring autophagy (3rd edition). Autophagy (2016) 12:1-222. doi:10.108 $0 / 15548627.2015 .1100356$

41. Settembre C, Fraldi A, Medina DL, Ballabio A. Signals from the lysosome: a control centre for cellular clearance and energy metabolism. Nat Rev Mol Cell Biol (2013) 14:283-96. doi:10.1038/nrm3565

42. Nazio F, Strappazzon F, Antonioli M, Bielli P, Cianfanelli V, Bordi M, et al. mTOR inhibits autophagy by controlling ULK1 ubiquitylation, self-association and function through AMBRA1 and TRAF6. Nat Cell Biol (2013) 15:406-16. doi:10.1038/ncb2708

43. Stolz A, Ernst A, Dikic I. Cargo recognition and trafficking in selective autophagy. Nat Cell Biol (2014) 16:495-501. doi:10.1038/ncb2979

44. Heo J-M, Ordureau A, Paulo JA, Rinehart J, Harper JW. The PINK1-PARKIN mitochondrial ubiquitylation pathway drives a program of OPTN/NDP52 recruitment and TBK1 activation to promote mitophagy. Mol Cell (2015) 60(1):7-20. doi:10.1016/j.molcel.2015.08.016

45. Redmann M, Dodson M, Boyer-Guittaut M, Darley-Usmar V, Zhang J. Mitophagy mechanisms and role in human diseases. Int J Biochem Cell Biol (2014) 53:127-33. doi:10.1016/j.biocel.2014.05.010

46. Westermann B. Mitochondrial fusion and fission in cell life and death. Nat Rev Mol Cell Biol (2010) 11(12):872-84. doi:10.1038/nrm3013

47. Deas E, Plun-Favreau H, Gandhi S, Desmond H, Kjaer S, Loh SH, et al. PINK1 cleavage at position A103 by the mitochondrial protease PARL. Hum Mol Genet (2011) 20:867-79. doi:10.1093/hmg/ddq526

48. Greene AW, Grenier K, Aguileta MA, Muise S, Farazifard R, Haque ME, et al. Mitochondrial processing peptidase regulates PINK1 processing, import and Parkin recruitment. EMBO Rep (2012) 13:378-85. doi:10.1038/ embor.2012.14

49. Narendra DP, Jin SM, Tanaka A, Suen DF, Gautier CA, Shen J, et al. PINK1 is selectively stabilized on impaired mitochondria to activate Parkin. PLoS Biol (2010) 8:e1000298. doi:10.1371/journal.pbio.1000298

50. Matsuda N, Sato S, Shiba K, Okatsu K, Saisho K, Gautier CA, et al. PINK1 stabilized by mitochondrial depolarization recruits Parkin to damaged mitochondria and activates latent Parkin for mitophagy. J Cell Biol (2010) 189:211-21. doi:10.1083/jcb.200910140 
51. Okatsu K, Oka T, Iguchi M, Imamura K, Kosako H, Tani N, et al. PINK1 autophosphorylation upon membrane potential dissipation is essential for Parkin recruitment to damaged mitochondria. Nat Commun (2012) 3:1016. doi:10.1038/ncomms2016

52. Kane LA, Lazarou M, Fogel AI, Li Y, Yamano K, Sarraf SA, et al. PINK1 phosphorylates ubiquitin to activate Parkin E3 ubiquitin ligase activity. J Cell Biol (2014) 205:143-53. doi:10.1083/jcb.201402104

53. Kazlauskaite A, Kelly V, Johnson C, Baillie C, Hastie CJ, Peggie M, et al. Phosphorylation of Parkin at Serine65 is essential for activation: elaboration of a MIRO1 substrate-based assay of Parkin E3 ligase activity. Open Biol (2014) 4:130213. doi:10.1098/rsob.130213

54. Koyano F, Okatsu K, Kosako H, Tamura Y, Go E, Kimura M, et al. Ubiquitin is phosphorylated by PINK1 to activate Parkin. Nature (2014) 510(7503):162-6. doi:10.1038/nature13392

55. Lazarou M, Sliter DA, Kane LA, Sarraf SA, Wang C, Burman JL, et al. The ubiquitin kinase PINK1 recruits autophagy receptors to induce mitophagy. Nature (2015) 524(7565):309-14. doi:10.1038/nature14893

56. Chen Y, Dorn GW. PINK1-phosphorylated mitofusin 2 is a Parkin receptor for culling damaged mitochondria. Science (2013) 340:471-5. doi:10.1126/ science. 1231031

57. Cunningham CN, Baughman JM, Phu L, Tea JS, Yu C, Coons M, et al. USP30 and Parkin homeostatically regulate atypical ubiquitin chains on mitochondria. Nat Cell Biol (2015) 17:160-9. doi:10.1038/ncb3097

58. Ordureau A, Sarraf SA, Duda DM, Heo JM, Jedrychowski MP, Sviderskiy VO, et al. Quantitative proteomics reveal a feedforward mechanism for mitochondrial PARKIN translocation and ubiquitin chain synthesis. $\mathrm{Mol}$ Cell (2014) 2:1-16. doi:10.1016/j.molcel.2014.09.007

59. Sarraf SA, Raman M, Guarani-Pereira V, Sowa ME, Huttlin EL, Gygi SP, et al. Landscape of the PARKIN-dependent ubiquitylome in response to mitochondrial depolarization. Nature (2013) 496:372-6. doi:10.1038/ nature 12043

60. Wang X, Winter D, Ashrafi G, Schlehe J, Wong YL, Selkoe D, et al. PINK1 and Parkin target miro for phosphorylation and degradation to arrest mitochondrial motility. Cell (2011) 147:893-906. doi:10.1016/j.cell.2011. 10.018

61. Geisler S, Holmström KM, Skujat D, Fiesel FC, Rothfuss OC, Kahle PJ, et al. PINK1/Parkin-mediated mitophagy is dependent on VDAC1 and p62/ SQSTM1. Nat Cell Biol (2010) 12:119-31. doi:10.1038/ncb2012

62. Sun Y, Vashisht AA, Tchieu J, Wohlschlegel JA, Dreier L. Voltage-dependent anion channels (VDACs) recruit Parkin to defective mitochondria to promote mitochondrial autophagy. J Biol Chem (2012) 287:40652-60. doi:10.1074/jbc. M112.419721

63. Wei Y, Chiang WC, Sumpter R, Mishra P, Levine B. Prohibitin 2 is an inner mitochondrial membrane mitophagy receptor. Cell (2016) 168(1-2):224-38. e10. doi:10.1016/j.cell.2016.11.042

64. Hollville E, Carroll RG, Cullen SP, Martin SJ. Bcl-2 family proteins participate in mitochondrial quality control by regulating Parkin/PINK1-dependent mitophagy. Mol Cell (2014) 55:451-66. doi:10.1016/j.molcel.2014.06.001

65. Strappazzon F, Cecconi F. AMBRA1-induced mitophagy: a new mechanism to cope with cancer? Mol Cell Oncol (2015) 2:e975647. doi:10.4161/237235 56.2014.975647

66. Bingol B, Tea JS, Phu L, Reichelt M, Bakalarski CE, Song Q, et al. The mitochondrial deubiquitinase USP30 opposes Parkin-mediated mitophagy. Nature (2014) 509:370-5. doi:10.1038/nature13418

67. Cornelissen T, Haddad D, Wauters F, Van Humbeeck C, Mandemakers W, Koentjoro B, et al. The deubiquitinase USP15 antagonizes Parkin-mediated mitochondrial ubiquitination and mitophagy. Hum Mol Genet (2014) 23:5227-42. doi:10.1093/hmg/ddu244

68. Durcan TM, Tang MY, Pérusse JR, Dashti EA, Aguileta MA, McLelland GL, et al. USP8 regulates mitophagy by removing K6-linked ubiquitin conjugates from Parkin. EMBO J (2014) 33:2473-91. doi:10.15252/embj.201489729

69. Van Humbeeck C, Cornelissen T, Hofkens H, Mandemakers W, Gevaert K, De Strooper B, et al. Parkin interacts with Ambral to induce mitophagy. J Neurosci (2011) 31:10249-61. doi:10.1523/JNEUROSCI.1917-11.2011

70. Strappazzon F, Nazio F, Corrado M, Cianfanelli V, Romagnoli A, Fimia GM, et al. AMBRA1 is able to induce mitophagy via LC3 binding, regardless of PARKIN and p62/SQSTM1. Cell Death Differ (2014) 22(3):419-32. doi: $10.1038 /$ cdd.2014.139
71. Wu W, Tian W, Hu Z, Chen G, Huang L, Li W, et al. ULK1 translocates to mitochondria and phosphorylates FUNDC1 to regulate mitophagy. EMBO Rep (2014) 15:566-75. doi:10.1002/embr.201438501

72. Chu CT, Ji J, Dagda RK, Jiang JF, Tyurina YY, Kapralov AA, et al. Cardiolipin externalization to the outer mitochondrial membrane acts as an elimination signal for mitophagy in neuronal cells. Nat Cell Biol (2013) 15:1197-205. doi: $10.1038 / \mathrm{ncb} 2837$

73. Toyama EQ, Herzig S, Courchet J, Lewis TL Jr, Losón OC, Hellberg K, et al. AMP-activated protein kinase mediates mitochondrial fission in response to energy stress. Science (2016) 351:275-81. doi:10.1126/science. aab4138

74. Liu L, Sakakibara K, Chen Q, Okamoto K. Receptor-mediated mitophagy in yeast and mammalian systems. Cell Res (2014) 24:787-95. doi:10.1038/ cr.2014.75

75. Zhang J, Ney PA. Role of BNIP3 and NIX in cell death, autophagy, and mitophagy. Cell Death Differ (2009) 16:939-46. doi:10.1038/cdd.2009.16

76. Sugiura A, McLelland G-L, Fon EA, McBride HM. A new pathway for mitochondrial quality control: mitochondrial-derived vesicles. EMBO J (2014) 33:1-15. doi:10.15252/embj.201488104

77. Sciacovelli M, Gonçalves E, Johnson TI, Zecchini VR, da Costa AS, Gaude $\mathrm{E}$, et al. Fumarate is an epigenetic modifier that elicits epithelial-to-mesenchymal transition. Nature (2016) 537(7621):544-7. doi:10.1038/nature19353

78. Zhao J, Zhang J, Yu M, Xie Y, Huang Y, Wolff DW, et al. Mitochondrial dynamics regulates migration and invasion of breast cancer cells. Oncogene (2013) 32:4814-24. doi:10.1038/onc.2012.494

79. Vyas S, Zaganjor E, Haigis MC. Mitochondria and cancer. Cell (2016) 166:555-66. doi:10.1016/j.cell.2016.07.002

80. Pinton P, Kroemer G. Cancer therapy: altering mitochondrial properties. Nat Chem Biol (2014) 10:89-90. doi:10.1038/nchembio.1440

81. Gaude E, Frezza C. Defects in mitochondrial metabolism and cancer. Cancer Metab (2014) 2:10. doi:10.1186/2049-3002-2-10

82. van Gisbergen MW, Voets AM, Starmans MH, de Coo IF, Yadak R, Hoffmann RF, et al. How do changes in the mtDNA and mitochondrial dysfunction influence cancer and cancer therapy? Challenges, opportunities and models. Mutat Res Rev Mutat Res (2015) 764:16-30. doi:10.1016/j. mrrev.2015.01.001

83. Warburg O. On the origin of cancer cells. Science (1956) 123:309-14. doi:10.1126/science.123.3191.309

84. Johnson RF, Perkins ND. Nuclear factor- $\kappa$ B, p53, and mitochondria: regulation of cellular metabolism and the Warburg effect. Trends Biochem Sci (2012) 37:317-24. doi:10.1016/j.tibs.2012.04.002

85. Ferri KF, Kroemer G. Organelle-specific initiation of cell death pathways. Nat Cell Biol (2001) 3:E255-63. doi:10.1038/ncb1101-e255

86. White E. The role for autophagy in cancer. J Clin Investig (2015) 125:42-6. doi:10.1172/JCI73941

87. Lock R, Roy S, Kenific CM, Su JS, Salas E, Ronen SM, et al. Autophagy facilitates glycolysis during Ras-mediated oncogenic transformation. Mol Biol Cell (2011) 22:165-78. doi:10.1091/mbc.E10-06-0500

88. Yang X, Yu DD, Yan F, Jing YY, Han ZP, Sun K, et al. The role of autophagy induced by tumor microenvironment in different cells and stages of cancer. Cell Biosci (2015) 5:14. doi:10.1186/s13578-015-0005-2

89. Pan H, Chen L, Xu Y, Han W, Lou F, Fei W, et al. Autophagy-associated immune responses and cancer immunotherapy. Oncotarget (2015) 7:2123546. doi:10.18632/oncotarget.6908

90. Galluzzi L, Pietrocola F, Bravo-San Pedro JM, Amaravadi RK, Baehrecke $\mathrm{EH}$, Cecconi F, et al. Autophagy in malignant transformation and cancer progression. EMBO J (2015) 34:856-80. doi:10.15252/embj.201490784

91. Pietrocola F, Pol J, Kroemer G. Fasting improves anticancer immunosurveillance via autophagy induction in malignant cells. Cell Cycle (2016) 15:3327-8. doi:10.1080/15384101.2016.1224797

92. Gomes LC, Di Benedetto G, Scorrano L. During autophagy mitochondria elongate, are spared from degradation and sustain cell viability. Nat Cell Biol (2011) 13:589-98. doi:10.1038/ncb2220

93. Chourasia AH, Boland ML, Macleod KF. Mitophagy and cancer. Cancer Metab (2015) 3:4. doi:10.1186/s40170-015-0130-8

94. Kryston TB, Georgiev AB, Pissis P, Georgakilas AG. Role of oxidative stress and DNA damage in human carcinogenesis. Mutat Res (2011) 711:193-201. doi:10.1016/j.mrfmmm.2010.12.016 
95. Grek CL, Tew KD. Redox metabolism and malignancy. Curr Opin Pharmacol (2010) 10:362-8. doi:10.1016/j.coph.2010.05.003

96. Maes H, Rubio N, Garg AD, Agostinis P. Autophagy: shaping the tumor microenvironment and therapeutic response. Trends Mol Med (2013) 19:428-46. doi:10.1016/j.molmed.2013.04.005

97. Bernardini JP, Lazarou M, Dewson G. Parkin and mitophagy in cancer. Oncogene (2016) 36(10):1315-27. doi:10.1038/onc.2016.302

98. Fujiwara M, Marusawa H, Wang HQ, Iwai A, Ikeuchi K, Imai Y, et al. Parkin as a tumor suppressor gene for hepatocellular carcinoma. Oncogene (2008) 27:6002-11. doi:10.1038/onc.2008.199

99. Pugh TJ, Morozova O, Attiyeh EF, Asgharzadeh S, Wei JS, Auclair D, et al. The genetic landscape of high-risk neuroblastoma. Nat Genet (2013) 45:279-84. doi:10.1038/ng.2529

100. Cianfanelli V, Fuoco C, Lorente M, Salazar M, Quondamatteo F, Gherardini $\mathrm{PF}$, et al. AMBRA1 links autophagy to cell proliferation and tumorigenesis by promoting c-Myc dephosphorylation and degradation. Nat Cell Biol (2015) 17:20-30. doi:10.1038/ncb3072

101. Chung KK, Thomas B, Li X, Pletnikova O, Troncoso JC, Marsh L, et al. S-nitrosylation of Parkin regulates ubiquitination and compromises Parkin's protective function. Science (2004) 304:1328-31. doi:10.1126/ science. 1093891

102. Laplante M, Sabatini DM. Regulation of mTORC1 and its impact on gene expression at a glance. J Cell Sci (2013) 126:1713-9. doi:10.1242/jcs.125773

103. Hanahan D, Weinberg RA. Hallmarks of cancer: the next generation. Cell (2011) 144:646-74. doi:10.1016/j.cell.2011.02.013

104. Wang L, Guo L, Lu L, Sun H, Shao M, Beck SJ, et al. Synaptosomal mitochondrial dysfunction in 5xFAD mouse model of Alzheimer's disease. PLoS One (2016) 11:e0150441. doi:10.1371/journal.pone.0150441

105. Saito Y, Chapple RH, Lin A, Kitano A, Nakada D. AMPK protects leukemia-initiating cells in myeloid leukemias from metabolic stress in the bone marrow. Cell Stem Cell (2015) 17:585-96. doi:10.1016/j.stem.2015.08.019

106. Martinez-Outschoorn UE, Pestell RG, Howell A, Tykocinski ML, Nagajyothi F, Machado FS, et al. Energy transfer in "parasitic" cancer metabolism: mitochondria are the powerhouse and Achilles' heel of tumor cells. Cell Cycle (2011) 10:4208-16. doi:10.4161/cc.10.24.18487

107. Capparelli C, Guido C, Whitaker-Menezes D, Bonuccelli G, Balliet R, Pestell TG, et al. Autophagy and senescence in cancer-associated fibroblasts metabolically supports tumor growth and metastasis, via glycolysis and ketone production. Cell Cycle (2012) 11:2285-302. doi:10.4161/cc.20718

108. Martinez-Outschoorn UE, Trimmer C, Lin Z, Whitaker-Menezes D, Chiavarina B, Zhou J, et al. Autophagy in cancer associated fibroblasts promotes tumor cell survival. Cell Cycle (2010) 9:3515-33. doi:10.4161/ cc.9.17.12928

109. Colombini M, Mannella CA. VDAC, the early days. Biochim Biophys Acta (2012) 1818:1438-43. doi:10.1016/j.bbamem.2011.11.014

110. Shoshan-Barmatz V, Mizrachi D. VDAC1: from structure to cancer therapy. Front Oncol (2012) 2:164. doi:10.3389/fonc.2012.00164

111. Mathupala SP, Ko YH, Pedersen PL. Hexokinase II: cancer's double-edged sword acting as both facilitator and gatekeeper of malignancy when bound to mitochondria. Oncogene (2006) 25:4777-86. doi:10.1038/sj.onc. 1209603

112. Chiara F, Castellaro D, Marin O, Petronilli V, Brusilow WS, Juhaszova M, et al. Hexokinase II detachment from mitochondria triggers apoptosis through the permeability transition pore independent of voltage-dependent anion channels. PLoS One (2008) 3:e1852. doi:10.1371/journal. pone. 0001852

113. Arif T, Vasilkovsky L, Refaely Y, Konson A, Shoshan-Barmatz V. Silencing VDAC1 expression by siRNA inhibits cancer cell proliferation and tumor growth in vivo. Mol Ther Nucleic Acids (2014) 3:e159. doi:10.1038/mtna.2014.9
114. Caino MC, Ghosh JC, Chae YC, Vaira V, Rivadeneira DB, Faversani A, et al. PI3K therapy reprograms mitochondrial trafficking to fuel tumor cell invasion. Proc Natl Acad Sci U S A (2015) 112:8638-43. doi:10.1073/ pnas. 1500722112

115. Cheng CT, Kuo CY, Ouyang C, Li CF, Chung Y, Chan DC, et al. Metabolic stress-induced phosphorylation of KAP1 Ser473 blocks mitochondrial fusion in breast cancer cells. Cancer Res (2016) 76:5006-18. doi:10.1158/0008-5472. CAN-15-2921

116. Guaragnella N, Giannattasio S, Moro L. Mitochondrial dysfunction in cancer chemoresistance. Biochem Pharmacol (2014) 92:62-72. doi:10.1016/j. bcp.2014.07.027

117. Mullen AR, Wheaton WW, Jin ES, Chen PH, Sullivan LB, Cheng T, et al. Reductive carboxylation supports growth in tumour cells with defective mitochondria. Nature (2011) 481(7381):385-8. doi:10.1038/nature10642

118. Rehman J, Zhang HJ, Toth PT, Zhang Y, Marsboom G, Hong Z, et al. Inhibition of mitochondrial fission prevents cell cycle progression in lung cancer. FASEB J (2012) 26:2175-86. doi:10.1096/ff.11-196543

119. Kashatus JA, Nascimento A, Myers LJ, Sher A, Byrne FL, Hoehn KL, et al. Erk2 phosphorylation of DRP1 promotes mitochondrial fission and MAPK-driven tumor growth. Mol Cell (2015) 57:537-51. doi:10.1016/j. molcel.2015.01.002

120. Ferreira-da-Silva A, Valacca C, Rios E, Pópulo H, Soares P, Sobrinho-Simões $\mathrm{M}$, et al. Mitochondrial dynamics protein DRP1 is overexpressed in oncocytic thyroid tumors and regulates cancer cell migration. PLoS One (2015) 10:e0122308. doi:10.1371/journal.pone.0122308

121. Senft D, Ronai ZA. Regulators of mitochondrial dynamics in cancer. Curr Opin Cell Biol (2016) 39:43-52. doi:10.1016/j.ceb.2016.02.001

122. Wan YY, Zhang JF, Yang ZJ, Jiang LP, Wei YF, Lai QN, et al. Involvement of DRP1 in hypoxia-induced migration of human glioblastoma U251 cells. Oncol Rep (2014) 32(2):619-26. doi:10.3892/or.2014.3235

123. Xie Q, Wu Q, Horbinski CM, Flavahan WA, Yang K, Zhou W, et al. Mitochondrial control by DRP1 in brain tumor initiating cells. Nat Neurosci (2015) 18:501-10. doi:10.1038/nn.3960

124. Campello S, Lacalle RA, Bettella M, Mañes S, Scorrano L, Viola A. Orchestration of lymphocyte chemotaxis by mitochondrial dynamics. J Exp Med (2006) 203:2879-86. doi:10.1084/jem.20061877

125. Da Silva AF, Mariotti FR, Máximo V, Campello S. Mitochondria dynamism: of shape, transport and cell migration. Cell Mol Life Sci (2014) 71:2313-24. doi:10.1007/s00018-014-1557-8

126. Desai SP, Bhatia SN, Toner M, Irimia D. Mitochondrial localization and the persistent migration of epithelial cancer cells. Biophys J (2013) 104:2077-88. doi:10.1016/j.bpj.2013.03.025

127. Töpfer K, Kempe S, Müller N, Schmitz M, Bachmann M, Cartellieri M, et al. Tumor evasion from T cell surveillance. J Biomed Biotechnol (2011) 2011:918471. doi:10.1155/2011/918471

128. Buck MD, O'Sullivan D, Klein Geltink RI, Curtis JD, Chang CH, Sanin $\mathrm{DE}$, et al. Mitochondrial dynamics controls $\mathrm{T}$ cell fate through metabolic programming. Cell (2016) 166:63-76. doi:10.1016/j.cell.2016.05.035

Conflict of Interest Statement: The authors declare that the research was conducted in the absence of any commercial or financial relationships that could be construed as a potential conflict of interest.

Copyright (C) 2017 Bordi, Nazio and Campello. This is an open-access article distributed under the terms of the Creative Commons Attribution License (CC BY). The use, distribution or reproduction in other forums is permitted, provided the original author(s) or licensor are credited and that the original publication in this journal is cited, in accordance with accepted academic practice. No use, distribution or reproduction is permitted which does not comply with these terms. 